\title{
Erratum
}

\section{Erratum: Jepson et al., "Spatially Patterned Electrical Stimulation to Enhance Resolution of Retinal Prostheses"}

In the article "Spatially Patterned Electrical Stimulation to Enhance Resolution of Retinal Prostheses" by Lauren H. Jepson, Paweł Hottowy, Keith Mathieson, Deborah E. Gunning, Władysław Dąbrowski, Alan M. Litke, and E. J. Chichilnisky, which appeared on pages 4871-4881 of the April 2, 2014 issue, a label in Figure 1E appeared incorrectly. "Current ( $\mu \mathrm{A})$ )" should instead appear as "pulse amplitude (pC)". This error does not affect the conclusions of the article. The article has been corrected online.

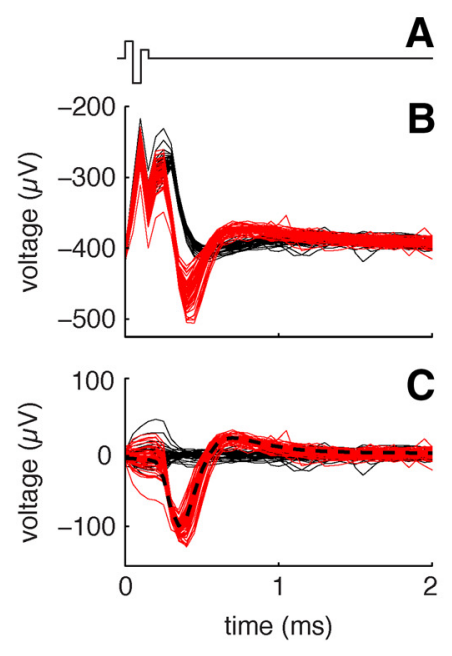

B
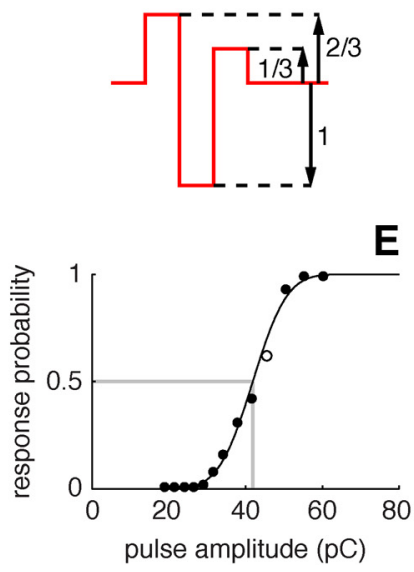

Figure 1.
D

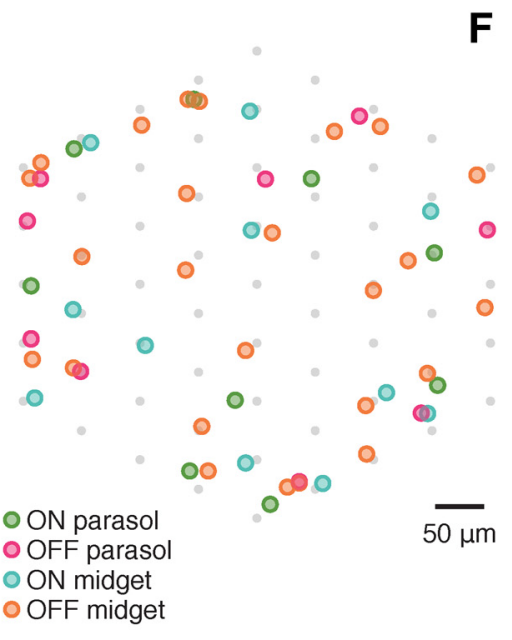

DOI: 10.1523/JNEUROSCI.0655-19.2019 